\title{
The use of transcriptomics to predict drug efficacy and treatment outcome in tuberculosis
}

\author{
Dimitrios Evangelopoulos \& Simon J Waddell
}

Several new drugs have recently been developed for the treatment of tuberculosis (TB) and, together with a number of repurposed drugs, are currently in clinical trials. The challenge remains to determine which of these novel compounds to prioritize, and to identify which drug combinations will be most effective. The task of testing each novel drug in combination with both new and existing antitubercular compounds using conventional methodologies is an expensive and daunting prospect. There is a need therefore for new assays to characterize drug efficacy, which are both robust and rapid, and which predict a superior regimen in combination drug therapy. In this chapter we discuss the use of gene expression profiling as a tool in the tuberculosis drug discovery pipeline, and the application of RNA-based assays for monitoring treatment success.

\section{Current tuberculosis drug discovery \& development}

The drug development pipeline for TB has been fuelled over the past 10 years by an explosion of new antitubercular chemical entities that have been dis- covered, or rediscovered as existing drugs are repurposed for TB. This is welcome and long-overdue since the last systematic introduction of TB- specific drugs was almost 50 years ago [1]. Many of the new drugs are undergoing clinical trials to evaluate their efficacy in combination therapies to treat fully drug-sensitive TB, and/or multi or extensively drug-resistant TB (MDR or XDR-TB). For upto-date information about the TB drug discovery pipeline visit the 'Working Group on New TB Drugs' website [2]. One of these new TB drugs, bedaquiline (formerly TMC207), has recently been approved by the US Food and Drug Administration (FDA) to be added to the treatment regimen of adult pulmonary MDR-TB. Furthermore, delamanid another novel chemical entity with antitubercular activity has been conditionally approved by the European Medicines Agency (EMA) for use in drug resistant TB.

Of the TB drugs that are currently in clinical trials, many are either new chemical entities derived from highthroughput whole cell screening assays, or are drugs that have not been traditionally used against TB but which are now being evaluated in combination with current anti-TB drugs (Table 6.1). For example, the nitroimidazol, pretomanid (PA-824), a new chemical entity currently under Phase III clinical trials, was discovered by screening a series of chemically-modified bicyclic nitroimidazoles derived originally from an anti-cancer hit molecule against Mycobacterium tuberculosis [3]. Pretomanid, with novel mechanism(s) of action is active against different physiological states of M. tuberculosis [4]. Early bactericidal activity (EBA) studies of pretomanid in combination with moxifloxacin and pyrazinamide showed good efficacy against drug-susceptible TB [5]. Furthermore, the same combination demonstrated significantly greater and faster bactericidal activity compared to standard antituberculosis treatment in phase Ilb trials [6]. In an alternative strategy, the fluoroquinolones exemplify an existing class of drugs that have been re-evaluated for their ability to kill M. tuberculosis. Fluoroquinolones are a family of antibiotics active against a broad-spectrum of bacteria including mycobacteria, which act by inhibiting DNA topoisomerase and DNA replication. The efficacy of moxifloxacin, an antibiotic from this group, was recently assessed in a phase III clinical trial, REMOxTB. This noninferiority clinical trial in patients with drug-susceptible M. tuberculosis was designed to identify whether substitution of either isoniazid or ethambutol for moxifloxacin was able to reduce treatment time from the current 6 months to a proposed 4 months [7]. Novel drug combinations from this clinical trial failed to pass the non-inferiority threshold highlighting that the addition of moxifloxacin to the current regimen is not enough to reduce the treatment duration to 4 months from the current requirement of 6 months [8]. Nevertheless, the REMOXTB trial demonstrated the successful use of a fluoroquinolone in TB treatment. Moxifloxacin is now part of the phase III trial, STAND, where a combination of pretomanid, moxifloxacin and pyrazinamide will be evaluated against both drug-susceptible and MDR-TB cases. 
Table 6.1. New possible additions to the antituberculosis drug portfolio.

\begin{tabular}{|c|c|c|c|c|}
\hline Drug & Existing drug class & $\begin{array}{l}\text { Mechanism of } \\
\text { action (MoA) }\end{array}$ & $\begin{array}{l}\text { Methods used for } \\
\text { elucidating MoA }\end{array}$ & Development stage \\
\hline $\begin{array}{l}\text { Pretomanid } \\
\text { (PA-824) }\end{array}$ & $\begin{array}{l}\text { New chemical entity } \\
\text { (NCE) }\end{array}$ & $\begin{array}{l}\text { Mycolic acid } \\
\text { biosynthesis, } \\
\text { respiratory chain, } \\
\text { multiple targets }\end{array}$ & $\begin{array}{l}\text { Transcriptomics and } \\
\text { metabolomics }\end{array}$ & Phase III \\
\hline Benzothiazinones & NCE & Arabinan synthesis & $\begin{array}{l}\text { Transcriptomics, } \\
\text { proteomics and } \\
\text { genetic mutation } \\
\text { studies }\end{array}$ & Preclinical \\
\hline Sutezolid & Oxazolidinone & $\begin{array}{l}\text { Protein synthesis; } \\
\text { 23S rRNA }\end{array}$ & $\begin{array}{l}\text { Cellular translation } \\
\text { assays }\end{array}$ & Phase II \\
\hline SQ109 & NCE & $\begin{array}{l}\text { Cell wall inhibition; } \\
\text { MmpL3 }\end{array}$ & $\begin{array}{l}\text { Genetic mutation } \\
\text { studies }\end{array}$ & Phase II \\
\hline Linezolid & Oxazolidinone & $\begin{array}{l}\text { Protein synthesis; } \\
235 \text { rRNA }\end{array}$ & Metabolomics & Phase II \\
\hline Rifapentine & Rifamycin & Translation & Metabolomics & $\begin{array}{l}\text { Phase II and Phase } \\
\text { III }\end{array}$ \\
\hline $\begin{array}{l}\text { Bedaquiline } \\
\text { (TMC207) }\end{array}$ & NCE & ATP synthesis & $\begin{array}{l}\text { Metabolomics, } \\
\text { genetic mutation } \\
\text { studies }\end{array}$ & $\begin{array}{l}\text { Phase II and Phase } \\
\text { III }\end{array}$ \\
\hline AZD5847 & Oxazolidinone & $\begin{array}{l}\text { Protein synthesis; } \\
\text { 23S rRNA }\end{array}$ & $\begin{array}{l}\text { Cellular translation } \\
\text { assays }\end{array}$ & Phase II \\
\hline $\begin{array}{l}\text { Delamanid } \\
\text { (OPC-67683) }\end{array}$ & NCE & $\begin{array}{l}\text { Mycolic acid } \\
\text { synthesis inhibition }\end{array}$ & Metabolomics & Phase III \\
\hline $\begin{array}{l}\text { Fluoroquinolones } \\
\text { (gatifloxacin and } \\
\text { moxifloxacin) }\end{array}$ & Repurposed & $\begin{array}{l}\text { DNA replication and } \\
\text { transcription; DNA } \\
\text { gyrase, } \\
\text { topoisomerase IV }\end{array}$ & $\begin{array}{l}\text { Genetic mutation } \\
\text { studies }\end{array}$ & Phase III \\
\hline Q203 & NCE & $\begin{array}{l}\text { ATP synthesis; } \\
\text { cytochrome bc1 } \\
\text { complex }\end{array}$ & $\begin{array}{l}\text { Genetic mutation } \\
\text { studies }\end{array}$ & Preclinical \\
\hline
\end{tabular}


TB is treated using combination therapy to achieve sterilization and to minimize the occurrence of drug resistance; the standard therapy regimen for drug-sensitive TB involves taking combinations of four drugs (isoniazid, rifampicin, ethambutol and pyrazinamide) over a period of 6 months. This provides a challenge for the design of clinical trials as new drugs need to be somehow prioritized and tested in novel combinations. Current solutions either modify standard TB chemotherapy by addition or replacement of an existing drug with a newly developed drug, or generate completely novel regimens. Innovative study design with multiarm, multistage trials where different combinations are tested alongside the standard treatment are also proposed [1]. Allied to the success of these trials is the ability to gauge the efficacy of new TB treatments in a short period of time. EBA studies measure the success of drug combinations by determining their bactericidal activity during the first two weeks of treatment. These studies rely on microbiological culture and therefore the readout can take up to 6 weeks due to the slow growth rate of M. tuberculosis. In addition, EBA studies of multiple combinations of drugs may be difficult to interpret; for example pyrazinamide, essential to the success of current regimens, does not have any antimicrobial effect in the first 2 days of treatment, but reduces bacterial numbers in sputum over the next 12 days [9]. Thus, there is an urgent need for new assays to monitor drug efficacy that may also help to characterize the action of novel drugs and drug combinations in vivo. Consortia such as PreDiCT-TB [10] are aiming to support this gap by generating and evaluating new decision making tools based on modelling in vitro, in vivo and clinical trials datasets to select successful future drug combinations. In such approaches, bacterial transcriptomics as unbiased assays are useful to map the changing state of TB bacilli after drug exposure.

\section{Transcriptomic analysis for understanding drug mode of action}

Many of the inhibitors currently under clinical development were identified using whole cell assays where the effect of compounds on bacterial killing is measured directly. This approach has proved particularly successful in mycobacterial research, where simply getting a novel chemical entity across the relatively impermeable mycobacterial cell wall is a triumph. However, the mechanism of action of these inhibitors at this stage is usually unknown. Understanding how a drug kills bacteria and identifying the molecular tar- get(s) is vital to improve drug efficacy and reduce cytotoxicity. In addition, understanding the mode of drug action also allows potential drug resistance mechanisms to be recognized, such as mutations to rpoB associated with rifampicin resistance. Two genome-wide methodologies are often employed to help define the class of action of a novel compound. The first is to identify mutations that lead to drug resistance; spontaneous drug-resistant bacterial mutants are generated in the laboratory and molecular methods employed to identify the mutated genes which may include the drug target. Nowadays, the genomes of resistant mutants are sequenced using next generation sequencing technologies to identify putative targets [11], or define factors that affect drug efficacy. However, this method is only feasible if resistant mutants can be successfully produced, which may not be possible for all categories of chemical [12]. In addition, the mutated gene(s) may not always determine the site of drug action, as in the case of prodrugs where often the activating enzyme is distinguished.

The second global approach, which is useful to apply before focused enzyme inhibition and drug-binding assays are developed for target definition, is transcriptional profiling the response of bacilli to new chemical entities. Transcriptome studies can provide useful insights into the target pathways affected by drug action, as well as decipher the differential regulation of processes that may influence drug efficacy. Conventionally, microarrays or qRT-PCR panels, and more recently RNAseq, have been employed to elucidate the class of action of novel inhibitors. Drugs with similar modes of action exert comparable stresses on bacilli which result in equivalent transcriptional responses to drug exposure. Thus, the transcriptional signature from a novel compound may be dissected by mapping the differentially expressed genes onto a genome-wide interpretive framework of transcriptional responses generated from the exposure of $M$. tuberculosis bacilli to numerous antimicrobials and inhibitors [13-15]. Evidence from such studies has aided the identification of targets for the benzothiazinones [16] and pretomanid [4]. In addition, meta-analysis of transcriptomics data derived from bacteria exposed to drugs alone or in combination may signpost the contribution of each constituent drug to the efficacy of that particular regimen. This approach has been applied to eukaryotes to generate a drug action network where the location of each drug in the network is dependent upon the drug-induced differential gene expression signature [17]. Using this net- work, the mode of action of a new compound can be identified alongside defining a new function to a previously studied drug. A similar methodology might also be applied to antitubercular drugs using the genomewide transcriptional datasets that are already available. 
Understanding the action of drugs in combination \& in vivo

Defining single drug action in vitro is a complex but well-trodden path; how- ever understanding how combinations of drugs act on bacilli residing in multiple physiological niches during infection is an enormous challenge. Bacteria in vivo likely adapt to the microenvironments that surround them, and this may lead to the generation of subpopulations of bacilli responding to different stimuli or growth conditions. These disparate bacterial phenotypic states may alter the ability of drugs to kill bacilli; specific bacterial populations may become exquisitely sensitive or tolerant to the action of a drug. For example, nonreplicating cells are likely to be tolerant to drugs that target processes which are not essential in nongrowing cells, such as DNA replication and cell wall biosynthesis pathways that are critically important in replicating bacilli. Different bacterial physiological states, generated in vitro and after infection, have been mapped extensively using transcriptional profiling [18-21]. Such mRNA profiling studies promise to reveal metabolic pathways that may be amiable to novel drug design, such as lipid metabolism pathways induced by TB bacilli in macrophages. Additionally, temporal patterns of gene expression have defined the transition between actively replicating mycobacterial populations and persister-like states $[22,23]$. Thus, it is possible (in some in vitro and animal model scenarios) to predict the tolerance of bacilli to drug exposure, and thus the efficacy of a drug on a specific population, from examining the bacterial transcriptional signature. For example, the lack of induction of INH-responsive genes in a murine model of infection after INH-exposure suggested that these bacilli were likely tolerant to isoniazid killing [24]. It is important therefore in novel drug discovery programs that several different models that capture the various physiological states of M. tuberculosis bacilli found in human disease (for example; actively replicating, viable but nonreplicating, low metabolic activity) are tested in order to comprehensively assay the potential impact of new inhibitors. Moreover, the characterization of bacilli during infection by transcriptional profiling also enables in vitro systems to be developed that test the efficacy of novel compounds in scenarios that mimic the multiple environments that may be encountered by bacilli during disease.

Another important issue that must be addressed in both preclinical and clinical studies is to recognize whether a new inhibitor will act well in combination with existing drugs and/or in new combinations of anti-TB drugs. In preclinical studies, this is usually tested using a synergism or checker-board assay where two drugs are tested in combination and their Fractional Inhibitory Concentration Index (FICl) is calculated. This class of assay determines whether there is a synergetic, an antagonistic or an indifferent effect in vitro. However, a synergistic in vitro effect may not be maintained in vivo, or an in vivo antagonistic effect may not be observed in vitro. For example, murine models of tuberculosis demonstrate antagonism between isoniazid and pyrazinamide, two firstline antituberculosis drugs, which was not identified in vitro since it is difficult to demonstrate pyrazinamide killing in most in vitro models [25]. Gene expression profiling may be utilized to define the efficacy of a drug combination by mapping the specific profile of a drug combination and comparing it to the gene profiles of each constituent drug. The presence of a drug-specific signature likely indicates some action of the drug, and a positive contribution to the regimen. Conversely, drugs that do not elicit a transcriptional response in combination therapy may not be enhancing bacterial killing in the specific model evaluated. A cancer study of lymphoma and tumour cells treated with a variety of anticancer drugs individually and in combination revealed, using RNAi technology, that targeting the expression of eight genes was sufficient to predict the mode of action of single drugs alone as well as their combinatory effect [26]. The same methodology was also able to recognize whether a drug combination represented an average of single drug effects, a signature dominated by one of the drugs, or a completely new profile uncovering a combinatory mode of action [26]. Thus, transcriptional profiling the effect of combinations of drugs in settings that mirror the mixed populations of bacteria found in vivo may highlight unseen synergetic or antagonistic effects between drugs targeting bacteria with equivalent or disparate phenotypes.

\section{Molecular markers for monitoring treatment success in tuberculosis}

It is vitally important to effectively and efficiently monitor responses to treatment during TB chemotherapy in order to successfully eradiate TB bacilli, avoid relapse and prevent the emergence of drug resistance. Furthermore, active treatment outcome measures give the physician the opportunity to adjust or enhance drug regimens on a patient-by-patient basis selecting the most appropriate combination of drugs. At present, the gold standard for TB treatment is liquid culture conversion after two months of chemotherapy, symbolising a target date where TB bacilli can no longer be cultured from patient sputum. In addition, smear microscopy and serial 
sputum colony counts are also employed for determining drug regimen efficacy. These classic microbiological measures currently act as predictive markers for treatment success. However, with the exception of smear microscopy, these methods require bacterial culture and because the growth rate of M. tuberculosis is slow, the turnaround time of these assays is long (weeks/months; 52 days for liquid MGIT cultures to be defined as negative). This restricts the ability of physicians to quickly amend treatment regimens and may influence treatment outcomes, especially when managing MDR-TB cases. Thus, new biomarkers are needed to monitor treatment and provide predictive outcomes soon after the start of chemotherapy, overcoming the limitations of the current methods where either the detection limit is low or the assay time is long. The lack of reliable prognostic biomarkers for drug efficacy and relapse is also hampering the design and performance of current clinical trials [1]. It is worth noting that individualized medicine for monitoring TB therapy is not likely to be relevant yet for the vast majority of TB patients who are in resource-poor settings. However, this approach is highly relevant to clinical trials where, for example, whole genome sequencing methods are employed to distinguish between patient relapse or reinfection. The discovery and development of indicative biomarkers will therefore help to support decision making policies in multi-regimen trials where the efficacy of novel drug combinations is under investigation.

The application of molecular-based culture-free assays would be a great advantage for monitoring treatment as the turnaround time should be significantly faster, meaning that results will be available sooner for health physicians to manage or start appropriate treatment [27]. These assays are usually PCR-based tests where the mycobacterial DNA or RNA is measured and converted into simple positive or negative scores. This is exemplified by the introduction of the GeneXpert MTB/RIF assay (Cepheid, Sunnyvale, CA) which has revolutionized the diagnosis of TB by detecting the rpoB gene from $M$. tuberculosis in sputum and bronchoalveolar lavage samples [28]; TB diagnosis being a distinct but related problem to measuring treatment outcomes. The GeneXpert MTB/RIF assay detects the presence of $M$. tuberculosis DNA in sputum samples as well as screening for rpoB gene mutations which are associated with rifampicin resistance and MDR-TB. The rapid $2 \mathrm{~h}$ assay time of this molecular method allows treatment to start directly after diagnosis and to be refined to the drug resistance background of the TB clinical isolate. This compares to classical microbiological-based drug susceptibility tests that may take over two weeks to obtain results. This time shift from 2 weeks or more to $2 \mathrm{~h}$ is possible due to the switch from microbiological to molecular PCR-based technologies. However, the application of the GeneXpert MTB/RIF assay to monitor treatment success is limited as mycobacterial DNA is a relatively stable molecule, which can remain in tissue months after bacterial sterilization [29] making DNA-based assays not widely applicable for treatment outcome measurements (Table 6.2).

Conversely, detection systems that target RNA, a labile and dynamic molecule, correlate positively with bacterial survival. Molecular assays that measure either TB ribosomal 16S rRNA or messenger RNA have both been used successfully to monitor early treatment success, to quantify bacterial loads, and to measure declining bacterial numbers during chemotherapy [30-32] (Table 6.2). The positive correlation between RNA detection/abundance and the number of viable bacteria as measured conventionally using colony forming units highlights these RNAbased assays as useful tools for preclinical drug discovery, and perhaps in the clinic for monitoring bacterial killing during TB chemotherapy. The advantages that these RNA-based detection assays have over conventional tests are that they are rapid and specific to $M$. tuberculosis because they do not depend on culturing mycobacteria or risk contamination; they also offer the potential to provide additional information about bacterial populations at the gene regulatory level. These culture-free methods provide meaningful information on treatment success by modeling the decline in bacterial load; correspondingly relapse may be identified before chemotherapy ends, providing an early opportunity to alter drug regimens accordingly. The application of global transcriptional profiling to TB bacilli derived from sputum before the start of chemotherapy [33] may enable RNA-based assays to be expanded to map gene expression signatures which predict drug efficacy by detecting drug tolerance or drug resistance patterns. In future, a panel of gene-specific assays may become useful markers for drug efficacy and treatment outcome. In addition, the use of global gene expression analyses as a tool in drug discovery from early preclinical research to clinical trials will enable new biomarkers to be characterized to evaluate drug efficacy, drug resistance, combinatory drug action and drug-drug interactions. 
Table 6.2. Comparison of molecular-based assays for monitoring TB treatment success.

\begin{tabular}{|c|c|c|c|}
\hline Assay & Molecule used for detection & Pros and cons ${ }^{\dagger}$ & Ref \\
\hline $\begin{array}{l}\text { PCR-based molecular } \\
\text { detection of nucleic acids } \\
\text { (DNA, RNA) }\end{array}$ & $\begin{array}{l}\text { 85B mRNA (fbpA ); 16S rRNA; } \\
\text { IS6110 (DNA) }\end{array}$ & $\begin{array}{l}\text { mRNA signal was undetectable } \\
\text { following two weeks of treatment; } \\
\text { 16S rRNA reduction was less than } \\
10 \text {-fold in the first } 10 \text { days; TB DNA } \\
\text { was often present following } \\
\text { successful treatment. }\end{array}$ & [32] \\
\hline mRNA detection & $\begin{array}{l}\text { Isocitrate lyase (icl); } \\
\text { fibronectin-binding protein } \\
\text { (fbpB); antigen 85B (fbpA); } \\
\text { alpha-crystalline homologue } \\
\text { (hspX); noncoding ribosomal } \\
\text { promoter region (rrnA-P1) }\end{array}$ & $\begin{array}{l}\text { icl mRNA transcript was detectable } \\
\text { after } 2 \text { months of chemotherapy; } \\
\text { however, it was not consistent when } \\
\text { compared with liquid and solid } \\
\text { culture outcomes at the same time- } \\
\text { points. }\end{array}$ & {$[31]$} \\
\hline $\begin{array}{l}\text { Molecular Bacterial Load } \\
\text { (MBL) assay }\end{array}$ & 16S rRNA & $\begin{array}{l}\text { Successful in quantifying bacterial } \\
\text { load longitudinally, correlated well } \\
\text { with liquid and solid culture data ( } 0 \text {, } \\
3,7,14 \text { and } 56 \text { days post- } \\
\text { chemotherapy). The incorporation of } \\
\text { an internal control helped to account } \\
\text { for performance inhibition. The limit } \\
\text { of detection defined to be } 100 \\
\text { bacteria per ml of sputum. }\end{array}$ & {$[30]$} \\
\hline GeneXpert MTB/RIF assay & rpoB gene (DNA) & $\begin{array}{l}\text { A fully automated rapid and robust } \\
\text { system that has been developed for } \\
\text { the diagnosis of TB; its application } \\
\text { for treatment monitoring is limited } \\
\text { as it measures genomic DNA. }\end{array}$ & [29] \\
\hline
\end{tabular}

+ An advantage of all molecular assays for treatment monitoring is that they are rapid in providing results; however, they often require specific expertise and they are more expensive compared to existing microbiological assays 


\section{Acknowledgements}

The authors would like to thank Professor Timothy D McHugh for commenting on the manuscript.

Financial \& competing interests disclosure

D Evangelopoulos and SJ Waddell are part of the PreDiCT-TB consortium (http://www.predict-tb.eu), which is funded from the Innovative Medicines Initiative Joint Undertaking (http://www.imi.europa.eu) under grant agreement No 115337, resources of which are composed of financial contribution from the European Union's Seventh Framework Programme (FP7/2007-2013) and EFPIA companies' in kind contribution. The authors have no other relevant affiliations or financial involvement with any organization or entity with a financial interest in or financial conflict with the subject matter or materials discussed in the manuscript apart from those disclosed. No writing assistance was utilized in the production of this manuscript. 
- There are a number of new chemical entities under clinical development for tuberculosis and prioritizing which of these will be tested, and in which drug combinations, in clinical trials remains a challenge.

- Transcriptional profiling has been a useful tool for discovering the mechanism of action of novel inhibitors, as well as characterizing and modeling the different mycobacterial physiological states in vitro and in vivo.

- Transcriptomic analyses may be useful in determining drug efficacy and in predicting the combinatory effects of multidrug regimens.

- As assay sensitivity improves, the detection of M. tuberculosis RNA offers a promising new class of biomarkers that may monitor treatment efficacy during TB chemotherapy.

Key terms

Treatment outcome measurement: a procedure for evaluating whether a treatment regime is successful in combating disease. In tuberculosis (TB) treatment, the measured parameters might be bacterial load assayed by microscopy or growth in liquid or solid media, clinical disease, morbidity and mortality, rate of reactivation or protective immunity. Common assays include sputum smear microscopy, two month sputum culture conversion, and the recently introduced, serial sputum colony counts.

TB combination therapy:

the use of more than one drug taken simultaneously to treat tuberculosis. The main advantage of combination therapy is to reduce the emergence of antibiotic resistance while sterilising effectively. The World Health Organization recommends a standard therapy for drug-sensitive TB which includes four months of isoniazid, rifampicin, ethambutol and pyrazinamide, followed by two months of isoniazid and rifampicin.

Transcriptional profiling:

the measurement of the relative abundance of mRNA molecules from a population of cells that represents a snapshot of bacterial adaptations to the microenvironment.

Early bactericidal activity (EBA):

monitoring the decline of the bacterial load in tubercular sputum after the start of chemotherapy; measured using microbiological methods. EBA studies are usually performed for up to two weeks following the beginning of treatment.

Fractional Inhibitory Concentration Index (FICI):

a measure of the effect of two drugs when compared with their single activities in vitro. The $\mathrm{FICl}$ is calculated by the sum of the ratios of the MIC for drug $A$ in presence of drug $B$ divided by the MIC of drug $A$, with the ratios of the MIC for drug $B$ in presence of drug $A$ divided by the MIC of drug $\mathrm{B}$. $\mathrm{FICl}$ values $\leq 0.5$ are classified as synergism, between 0.5 and 4.0 as no interaction or indifferent, and $\geq 4.0$ as antagonism between drugs $A$ and $B$. 


\section{References}

1. Zumla A, Nahid P, Cole ST. Advances in the development of new tuberculosis drugs and treatment regimens. Nat. Rev. Drug Discov. 12(5), 388-404 (2013).

2. Working Group on New TB Drugs: Drug Pipeline. http://www.newtbdrugs.org/pipeline.php

3. Stover CK, Warrener P, Vandevanter DR et al. A small-molecule nitroimidazopyran drug candidate for the treatment of tuberculosis. Nature 405(6789), 962-966 (2000).

4. Manjunatha $\mathrm{U}$, Boshoff $\mathrm{HI}$, Barry $\mathrm{CE}$. The mechanism of action of PA-824: novel insights from transcriptional profiling. Comm. Integrative Biol. 2(3), 215-218 (2009).

5. Diacon AH, Dawson R, Von Groote-Bidlingmaier F et al. 14-day bactericidal activity of PA-824, bedaquiline, pyrazinamide, and moxifloxacin combinations: a randomised trial. Lancet 380(9846), 986-993 (2012).

6. Dawson R, Diacon AH, Everitt $D$ et al. Efficiency and safety of the combination of moxifloxacin, pretomanid (PA-824), and pyrazinamide during the first 8 weeks of antituberculosis treatment: a phase $2 \mathrm{~b}$, open-label, partly randomised trial in patients with drug-susceptible or drug-resistant pulmonary tuberculosis. Lancet 385 (9979), 1738-1747 (2015).

7. Trial ID: NCT00864383. http://clinicaltrials.gov

8. Gillespie SH, Crook AM, McHugh TD et al. Four-month moxifloxacin-based regimens for drug-sensitive tuberculosis. New England J. Med. 371(17), 1577-1587 (2014).

9. Mitchison D, Davies G. The chemotherapy of tuberculosis: past, present and future. Int. J. Tuberc. Lung Dis. 16(6), 724-732 (2012).

10. PreDiCT-TB consortium. http://www.predict- tb.eu

11. Andries $\mathrm{K}$, Verhasselt $\mathrm{P}$, Guillemont $\mathrm{J}$ et al. A diarylquinoline drug active on the ATP synthase of Mycobacterium tuberculosis. Science 307(5707), 223-227 (2005).

12. Gold B, Pingle M, Brickner SJ et al. Nonsteroidal anti-inflammatory drug sensitizes Mycobacterium tuberculosis to endogenous and exogenous antimicrobials. Proc. of Natl Acad. Sci. USA 109(40), 16004-16011 (2012).

13. Boshoff HI, Myers TG, Copp BR, Mcneil MR, Wilson MA, Barry CE 3rd. The transcriptional responses of Mycobacterium tuberculosis to inhibitors of metabolism: novel insights into drug mechanisms of action. J. Biol. Chem. 279(38), 40174-40184 (2004).

14. Waddell SJ, Stabler RA, Laing K, Kremer L, Reynolds RC, Besra GS. The use of microarray analysis to determine the gene expression profiles of Mycobacterium tuberculosis in response to anti-bacterial compounds. Tuberculosis (Edinb.) 84(3-4), 263-274 (2004).

15. Betts JC, Mclaren A, Lennon MG et al. Signature gene expression profiles discriminate between isoniazid-, thiolactomycin-, and triclosan-treated Mycobacterium tuberculosis. Antimicrob. Agents Chemother. 47(9), 29032913 (2003).

16. Makarov V, Manina G, Mikusova $\mathrm{K}$ et al. Benzothiazinones kill Mycobacterium tuberculosis by blocking arabinan synthesis. Science 324(5928), 801-804 (2009).

17. Iorio $F$, Bosotti $R$, Scacheri $E$ et al. Discovery of drug mode of action and drug repositioning from transcriptional responses. Proc. of Natl Acad. Sci. USA 107(33), 14621-14626 (2010).

18. Deb C, Lee CM, Dubey VS et al. A novel in vitro multiple-stress dormancy model for Mycobacterium tuberculosis generates a lipid-loaded, drug-tolerant, dormant pathogen. PLoS ONE 4(6), e6077 (2009).

19. Rohde KH, Veiga DF, Caldwell S, Balazsi G, Russell DG. Linking the transcriptional profiles and the physiological states of Mycobacterium tuberculosis during an extended intracellular infection. PLoS Pathogens 8(6), e1002769 (2012).

20. Waddell S. Reprogramming the Mycobacterium tuberculosis transcriptome during pathogenesis. Drug Discov. Today 7(1), e67-e73 (2010).

21. Galagan J E, Minch K, Peterson M et al. The Mycobacterium tuberculosis regulatory network and hypoxia. Nature 499 (7457), 178-183 (2013).

22. Keren I, Minami S, Rubin E, Lewis K. Characterization and transcriptome analysis of Mycobacterium tuberculosis persisters. mBio 2(3), e00100-00111 (2011).

23. Rustad TR, Harrell MI, Liao R, Sherman DR. The enduring hypoxic response of Mycobacterium tuberculosis. PLoS ONE 3(1), e1502 (2008).

24. Karakousis PC, Williams EP, Bishai WR. Altered expression of isoniazid-regulated genes in drug-treated dormant Mycobacterium tuberculosis. J. Antimicrob. Chemother. 61(2), 323-331 (2008). 
25. Grosset J, Almeida D, Converse PJ et al. Modeling early bactericidal activity in murine tuberculosis provides insights into the activity of isoniazid and pyrazinamide. Proc. of Natl Acad. Sci. USA 109(37), 15001-15005 (2012).

26. Pritchard JR, Bruno PM, Gilbert LA, Capron KL, Lauffenburger DA, Hemann MT. Defining principles of combination drug mechanisms of action. Proc. of Natl Acad. Sci. USA 110(2), E170-179 (2013).

27. Lohmann S, Herold A, Bergauer $T$ et al. Gene expression analysis in biomarker research and early drug development using function tested reverse transcription quantitative real-time PCR assays. Methods 59(1), 10-19 (2013).

28. Boehme CC, Nabeta P, Hillemann D et al. Rapid molecular detection of tuberculosis and rifampin resistance. N. Engl. J. Med. 363(11), 1005-1015 (2010).

29. Kayigire XA, Friedrich SO, Venter A et al. Direct comparison of Xpert MTB/RIF assay with liquid and solid mycobacterial culture for quantification of early bactericidal activity. J. Clin. Microbiol. 51(6), 1894-1898 (2013).

30. Honeyborne I, Mchugh TD, Phillips PP et al. Molecular bacterial load assay, a culture-free biomarker for rapid and accurate quantification of sputum Mycobacterium tuberculosis bacillary load during treatment. J. Clin. Microbiol. 49(11), 3905-3911 (2011).

31. Li L, Mahan CS, Palaci M et al. Sputum Mycobacterium tuberculosis mRNA asa marker of bacteriologic clearance in response to antituberculosis therapy. J. Clin. Microbiol. 48(1), 46-51 (2010).

32. Desjardin LE, Perkins MD, Wolski K et al. Measurement of sputum Mycobacterium tuberculosis messenger RNA as a surrogate for response to chemotherapy. Am. J. of Respir. Crit. Care Med. 160(1), 203-210 (1999).

33. Garton NJ, Waddell SJ, Sherratt AL et al. Cytological and transcript analyses reveal fat and lazy persister-like bacilli in tuberculous sputum. PLoS Med. 5(4), e75 (2008). 